\title{
Asymmetric Phase Transition Pathways During Li/Na Migration in 2D Materials
}

\author{
Shulin Chen ${ }^{1,2}$, Peng Gao ${ }^{2,3,4}$ \\ 1. State Key Laboratory of Advanced Welding and Joining, Harbin Institute of Technology, Harbin, \\ China \\ 2. Electron microscopy laboratory, School of Physics, and Center for Nanochemistry, Peking University, \\ Beijing, China \\ 3. Collaborative Innovation Center of Quantum Matter, Beijing, China \\ 4. Center for Nanochemistry, Peking University, Beijing, China
}

For lithium ion batteries, one of the fundamental issues is the energy efficiency, mainly influenced by the voltage hysteresis between the charge and discharge curves obtained during galvanostatic tests. The voltage hysteresis is expected in all different types of electrode materials with different origins. For the conversion, alloying and displacement electrode materials, the voltage hysteresis is usually expected from the equilibrium phase diagram. When the temperature is relatively low, the phase transition usually follows the nonequilibrium path and therefore structures prefer to convert into the phase that is the most easily kinetically reached instead of the thermodynamic ground state. The kinetic phase sequence, unlike the thermodynamic phase transition, is not reversible during cycling, leading to the voltage hysteresis (phase hysteresis) and low energy efficiency. In fact, in the conversion electrodes, the large voltage hysteresis during cycling is one of the main issues that hamper their commercialization. In the intercalation electrodes, the symmetric reaction pathways with opposite directions during charge and discharge are usually expected. The origin of the voltage hysteresis is believed to be the fact that the particles do not react with lithium simultaneously [1]. However, the kinetic processes in practical batteries are difficult to extract from the microscopic measurements due to the heterogeneous reactions in an electrode with millions of nanostructured units.

Here, we study the reaction pathways during $\mathrm{Li} / \mathrm{Na}$ intercalation and de-intercalation in the layered metal dichalcogenides by using in situ high-resolution transmission electron microscopy [2-4]. We find that the $\mathrm{Li} / \mathrm{Na}$ intercalation in the $\mathrm{SnS}_{2}$ occurs via a fast two-phase reaction, which is initiated by nucleation of $\mathrm{LiSnS}_{2} / \mathrm{NaSnS}_{2}$ with expanded lattice and richness of defects and followed by propagation of the phase boundary in Figure 1a. However, the $\mathrm{Li} / \mathrm{Na}$ de-intercalation involves intermediate phases with local structure ordering (i.e., $\mathrm{Li}_{0.5} \mathrm{SnS}_{2}$ and $\mathrm{Na}_{0.5} \mathrm{SnS}_{2}$ ) instead of retracting the original phase boundary, being solid-solution-like in Figure 1b. These $\mathrm{Li}_{0.5} \mathrm{SnS}_{2} / \mathrm{Na}_{0.5} \mathrm{SnS}_{2}$ domains with a few nanometers in size nucleate rapidly and heterogeneously upon $\mathrm{Li} / \mathrm{Na}$ extraction. The atomic structures of the intermediate superstructure $\left(\mathrm{Li}_{0.5} \mathrm{SnS}_{2} / \mathrm{Na}_{0.5} \mathrm{SnS}_{2}\right)$ have also been determined. These phases have alternative $\mathrm{Li} / \mathrm{Na}$ and vacancies rows along [110] direction within every (001) Li/Na plane, which is distinct from the common staging phase that consists of $\mathrm{Li} / \mathrm{Na}$ and vacancy (001) planes. Combining with density functional theory calculations, we conclude that this intermediate phase with local structure ordering in the layered structure is kinetically favored and structurally stable.

The distinct lithium insertion and extraction reaction pathways in the intercalation-type of electrodes (Figure 1c) are in contrast to the general two-phase reaction mode. Such asymmetric reaction pathways have also been proposed in other van der Waals intercalation materials such as $\mathrm{MoS}_{2}$. However, from the previous X-ray diffraction measurements [5], the details of intermediate and local structure evolution and asymmetric reaction pathways still remained unclear. Considering the structural similarities between 
$\mathrm{SnS}_{2}$ and $\mathrm{MoS}_{2}$, the intermediate phase with local structure ordering during $\mathrm{Li} / \mathrm{Na}$ extraction may also exist in $\mathrm{MoS}_{2}$ and other layered metal dichalcogenides with van der Waals interactions.

In summary, these findings provide valuable insights into the mechanistic understanding of the underlying electrochemistry in the van der Waals interactions electrode materials. The unexpected intermediate phases with local structure ordering are observed in the layered metal dichalcogenides in real-time and high-resolution. The atomic structure and dynamic behaviors are determined. The unusual row-ordering rather than the regular staging phases with plane-ordering has not been observed before. Such asymmetric reaction pathways are usually not expected in the intercalation electrode materials, which can also help us to elucidate the origins of voltage hysteresis in intercalation electrodes and thus shed light on design of high-energy efficiency batteries [6].

\section{References:}

[1] W. Dreyer et al, Nat Mater. 9 (2010), p. 448.

[2] P. Gao et al, Nano Lett. 16 (2016), p. 5582.

[3] P. Gao et al, Nano Energy 32 (2017), p. 302.

[4] P. Gao et al, ACS Nano 9 (2015), p. 11296.

[5] X. Wang et al, ACS Nano 8 (2014), p. 11394.

[6] The authors gratefully acknowledge the financial support through the National Natural Science Foundation of China (51502007, 51502032, 51522201), National Basic Research Program of China (2016YFA0300903), the National Program for Thousand Young Talents of China, and "2011 Program" Peking- Tsinghua-IOP Collaborative Innovation Center of Quantum Matter.
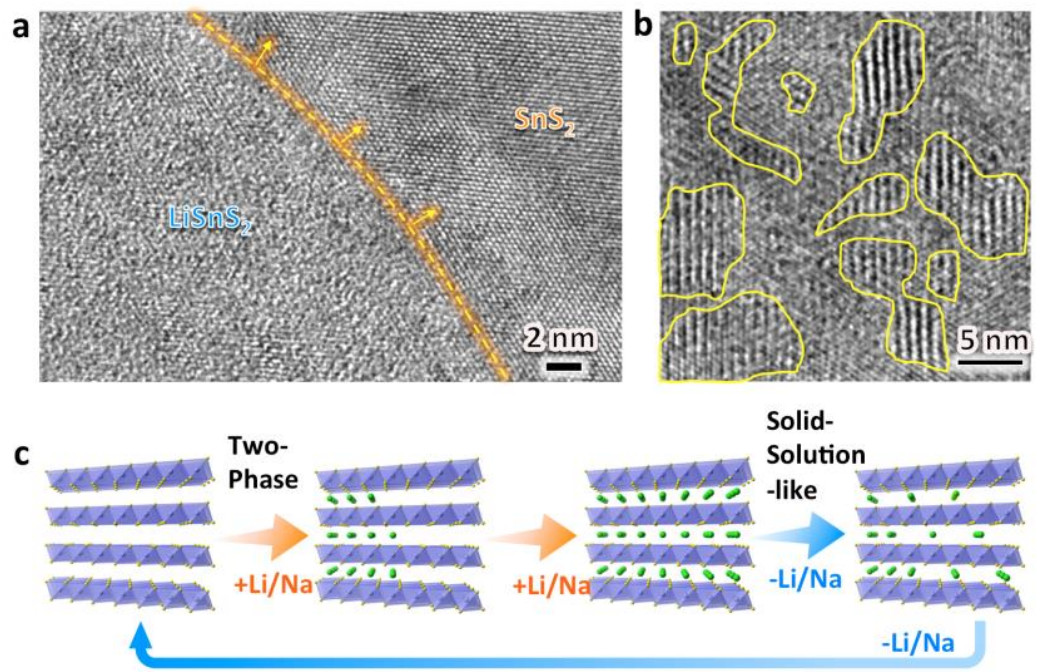

Figure 1. (a) Lithium insertion in $\mathrm{SnS}_{2}$ nanostructures via two-phase reactions. The viewing direction is [111]. The yellow dashed line highlights the reaction front (phase boundary). The arrows indicate the reaction front motion direction. (b) Lithium extraction from the $\mathrm{SnS}_{2}$ nanostructures involves intermediate $\mathrm{Li}_{0.5} \mathrm{SnS}_{2}$ phase with local structure ordering stripes. The yellow circles outline the intermediate domains. (c) Schematic showing the asymmetric reaction pathways during lithium insertion and extraction. 